\title{
Cr:LiSAF Slab Laser Performance
}

\author{
F. Hanson
}

\begin{abstract}
The performance of a flash-lamp pumped $\mathrm{Cr}^{3+}$ : LiSrAlF $_{6}$ multi-bounce slab laser is reported. The slab was conductively-cooled by a sapphire window which also transmitted the pump light. Laser output of $9 \mathrm{~mJ}$ and a slope efficiency of $0.061 \%$ were obtained although less than $15 \%$ of the total pump aperture was used.
\end{abstract}

\section{INTRODUCTION}

$\mathbf{T}$ THERE has been considerable activity recently in developing $\mathrm{Cr}^{3+}: \mathrm{LiSrAlF}_{6}$ (Cr:LiSAF) lasers for various applications in the near-IR. This material has two broad absorption bands centered at 450 and $650 \mathrm{~nm}$ and a suitably long excited-state lifetime, all of which allows for efficient flash-lamp pumping [1]. The broad gain spectrum from less than $800 \mathrm{~nm}$ to greater than $1000 \mathrm{~nm}$ allows wide tunability and efficient generation or amplification of very short optical pulses [2]. The potential also exists for a relatively simple all solid-state blue-green source using laser diode pumping and straight-forward second harmonic generation.

One of the problems encountered in scaling the average power output from Cr:LiSAF lasers has been the poor thermal conductivity and fracture toughness of the material [3]-typical of many fluoride crystal hosts. In order to alleviate this problem, one must reduce the pump-induced heating and/or use a geometry with increased surface area to improve the thermal transport. Recent demonstrations of efficient laser action with highly-doped Cr:LiSrAlF 6 pumped at $752 \mathrm{~nm} \mathrm{[4]}$ and with the stoichiometric material $\mathrm{LiSrCrF}_{6}$ pumped from $770 \mathrm{~nm}$ to $790 \mathrm{~nm}$ in the long wavelength tail of the absorption band [5], have shown that it would be feasible to use AlGaAs laser diodes as pump sources. Pumping in this wavelength region, as opposed to broad band flash-lamp pumping, would dramatically reduce the quantum defect between the excitation and emission photon energy. In addition, there are improvements in average power capability that could be realized by using a high aspect ratio slab geometry instead of a more conventional rod geometry [6]. The present work investigates the performance of a flash-lamp pumped Cr:LiSAF slab-laser in a multi-bounce active-mirror configuration. To the author's knowledge, there have been no published reports of Cr:LiSAF lasers using this approach.

\section{EXPERIMENTAL}

The laser experiments were performed with the same flashlamp pump head that was used earlier to measure gain in the slab geometry [7]. A 4-mm bore lamp with a 2.5-in arc

Manuscript received September 24, 1994; revised May 2, 1994.

The author is with the Naval Command, Control, and Ocean Surveillance Center, Code 843, San Diego, CA 92152-5732 USA.

IEEE Log Number 9409197.

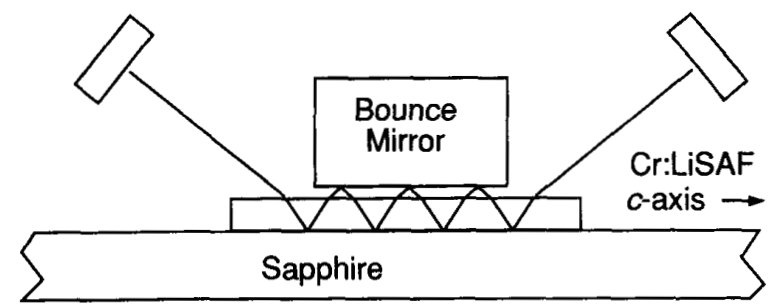

Fig. 1. Experimental set-up for conductively-cooled, multi-bounce slab laser.

length was used. A single 0.125 -in thick, rectangular sapphire window was mounted on one side of the head over an open aperture $0.6 \times 6.5 \mathrm{~cm}$ while a piece of silver foil was placed on the opposite side of the lamp. Cooling water at $T=20^{\circ} \mathrm{C}$ flowed around the lamp and in the $\sim 2 \mathrm{~mm}$ gap between the lamp and the window. A small glass slide with a transmission cut-off below $300 \mathrm{~nm}$ was placed in the coolant passage between the window and the flash-lamp. The Cr:LiSAF slab with 16 atomic-\% doping and measuring $18.6 \times 9.0 \times 1.6 \mathrm{~mm}$ was cemented with UV-cured epoxy to the outside of the sapphire window, which served both as a heat sink and a transparent window for the pump light. The two large faces of the slab were highly polished and a dielectric coating, designed to give high reflection for $p$-polarized light at $54^{\circ}$ incidence and $\lambda=850 \mathrm{~nm}$, was applied to one face of the slab. The coated side of the slab was cemented onto the sapphire window. The external bounce-mirror with the same dielectric coating was placed adjacent and parallel to the uncoated slab surface so that the cavity path consisted of multiple bounces between the highly reflecting surfaces of the slab and bouncemirror as shown in Fig. 1. This mirror was a rectangular piece that was cut from a larger round mirror such that the dielectric coating was present up to the edge. There were irregularities of $\sim 0.1 \mathrm{~mm}$ in the sawed edge. The angle of incidence was chosen to be near Brewster's angle to minimize the reflection loss on the uncoated slab surface. In this geometry, the gain is maximized when the $c$-axis of the Cr:LiSAF is parallel to the reflecting face of the slab and the plane of incidence. This was along the long dimension of the slab and parallel to the flash-lamp axis.

Adjustment of the spacing between the bounce-mirror and the slab affects the beam aperture, the number of bounces, and the total volume of the Cr:LiSAF slab available for energy extraction. The horizontal beam aperture $s$ (in the bounce plane) is given by

$$
s=2\left(h \sin \left(\theta_{e}\right)+d \cos \left(\theta_{e}\right) \tan \left(\theta_{i}\right)\right),
$$

where $d=1.6 \mathrm{~mm}$ is the slab thickness, $h$ is the mirror spacing, and $\theta_{e}\left(\theta_{i}\right)$ is the external (internal) angle of incidence. In 


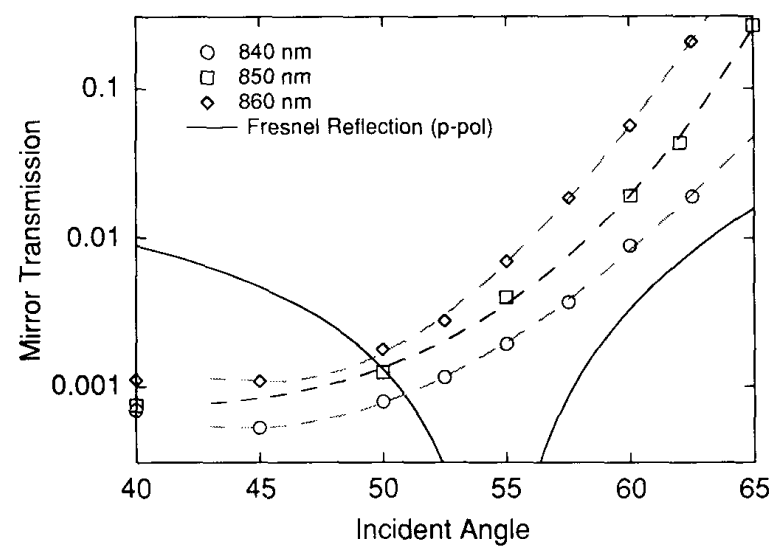

Fig. 2. Measured p-polarized transmission of the mirror coating applied to one side of the slab and the bounce-mirror. Also, the calculated Fresnel reflection for $n=1.4$

general, for a given length of bounce-mirror, there will be a discontinuous break inside the beam aperture where one part will experience one less bounce than the other part. This will lead to additional diffraction losses depending on the $\mathrm{TEM}_{00}$ mode size in the cavity. If the mirror length, $x$ is chosen such that

$$
x=2 m\left(h \tan \left(\theta_{e}\right)+d \tan \left(\theta_{i}\right)\right),
$$

the entire beam will experience $m$ bounces without a discontinuity. The out-of-plane beam aperture is not affected by the bounce-mirror and is only limited by the slab width and pump distribution. In the experiments described below, the end mirrors were a $30-\mathrm{cm}$ radius high reflector and a flat output coupler and the total cavity length was $\sim 15 \mathrm{~cm}$. The slab was placed equidistant from the two mirrors. The resulting mode $\mathrm{TEM}_{00}$ diameter at the slab was $\sim 240 \mu \mathrm{m}$ with a very small amount of astigmatism due to the path through the slab at non-normal incidence.

The laser performance was expected to depend critically on the quality of the high-reflecting dielectric coating applied to the slab and bounce-mirror. A Ti:sapphire laser was used to measure the p-polarized transmission of the mirror coating over a range of angles for $\lambda=840,850$, and $860 \mathrm{~nm}$. The results are given in Fig. 2 . The coating on the slab should show essentially the same transmission behaviour for light incident from the slab interior after conversion to the appropriate internal refracted angle. The calculated Fresnel reflection for $n=1.4$ is also plotted on the same scale. Ideally, the minimum of the coating transmission and the Fresnel reflection would coincide at the Brewster angle of $\sim 54^{\circ}$. From these measurements however, it was expected that the laser efficiency would be optimized at an incident angle closer to $50^{\circ}$. At normal incidence, the dielectric coating was reasonably transparent to the flash-lamp light from $400-800 \mathrm{~nm}$, including the range of the strongest Cr:LiSAF pump bands.

The slab-laser was aligned with a bounce-mirror $9.7 \mathrm{~mm}$ long and separated from the slab by $\sim 1 \mathrm{~mm}$. Initially, the reflection angle was set to $\sim 50^{\circ}$. A noticeable variation in the laser output was observed as the bounce-mirror was translated

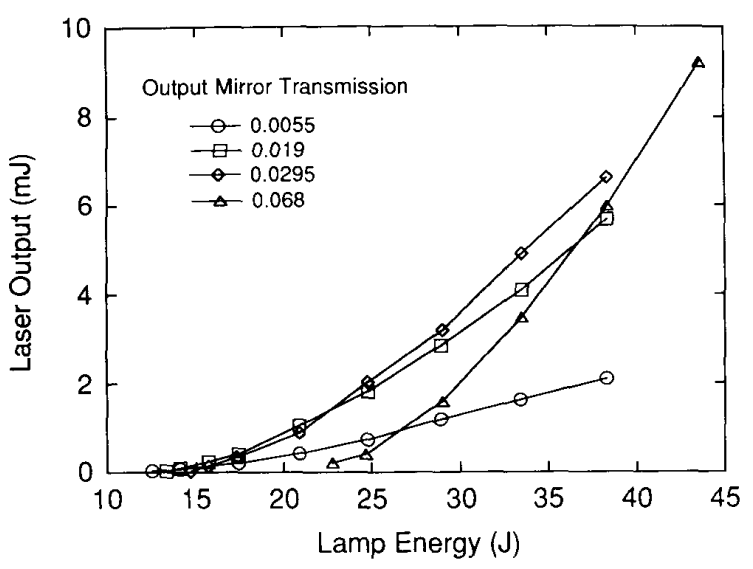

Fig. 3. Laser output measured for different output-coupling mirrors.

TABLE I

Measured Threhsold Energies and Slope Efficiencies fOR DifFerent OUtPUT Mirror Transmissions

\begin{tabular}{cccc}
\hline Mirror Transmission & Pump Threshold $(\mathrm{J})$ & Slope Efficiency $(\mathrm{mJ} / \mathrm{J})$ \\
\hline 0.0055 & 11.4 & 0.104 \\
0.019 & 12.9 & 0.301 \\
0.0295 & 14.5 & 0.368 \\
0.068 & 20.8 & 0.609 \\
\hline
\end{tabular}

normal to the slab face. A local optimum was found for $h \sim 0.5 \mathrm{~mm}$. According to (2), this gave a mode with three reflections from the bounce-mirror and 4 reflections from the coated slab face. The geometric beam aperture $s$ is calculated to be $2.1 \mathrm{~mm}$. No significant dependence of laser output with reflection angle was observed.

\section{LASER PERformanCE}

The laser energies shown in Fig. 3 were measured with a pyroelectric detector (Laser Precision model RiP-735) using 4 different output coupling mirrors. The flash-lamps were operated at $4-\mathrm{Hz}$ and the pulse wave form was $52 \mu$ s fullwidth at half-maximum. A red cut-off filter was used to block most of the flash-lamp light from reaching the detector and the remaining leakage was accounted for. The temporal output generally consisted of intense relaxation oscillations separated by a few $\mu$ s. The laser performance was characterized by analysis of the pump thresholds and slope efficiencies and the relevant data is summarized in Table I. Transmission data for all of the mirrors were measured on a Varian 2390 spectrophotometer and the values for $\lambda=850 \mathrm{~nm}$ are given. The mirror transmission did not vary significantly over the $\sim 10 \mathrm{~nm}$ bandwidth of laser emission. At threshold, the roundtrip gain $g=2\left[n^{*}\right] \sigma L$ is equated to the combined loss due to mirror transmission $T$ and various absorption and scattering losses $\alpha$, to give

$$
P_{t h}=-\frac{1}{2 \eta_{p} \sigma L}(\ln (1-T)+\ln (1-\alpha)) .
$$




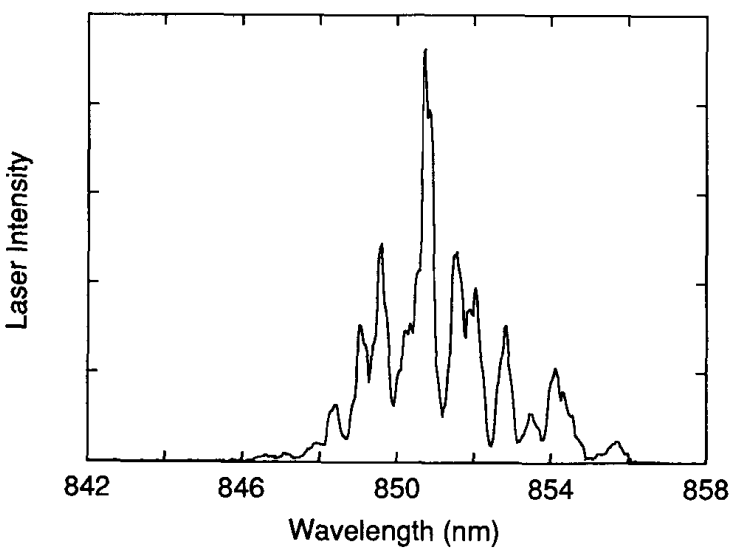

Fig. 4. Laser emission spectrum measured at $33 \mathrm{~J}$ with the $T=6.8 \%$ output mirror.

Here, $\sigma$ is the effective emission cross section and $L$ is the zigzag path length through the slab. We have defined a pump efficiency $\eta_{p}=\left[n^{*}\right] / P$, in terms of the peak inversion density $\left[n^{*}\right]$ averaged over the path, to include factors such as pump light absorption and excited state decay during the pump pulse. Generally, $\eta_{p}$ is assumed to be independent of pump energy, and $\ln (1-\alpha)$ and $2 \eta_{p} \sigma L$ are obtained by linear regression. For the data reported here, there is a slight but noticeable upwards curvature which is evidently due to increased upconversion losses [8] at higher pump levels. We obtain a loss $\alpha=0.081$ by using the data for the lower three values of output mirror transmission. The measured threshold at the highest output coupling, $T=0.068$, was then $\sim 2 \mathrm{~J}$ higher than predicted which is consistent with our earlier measurements of small signal gain, where we observed a roll-off of $\sim 10 \%$ at $20 \mathrm{~J}$. The relatively high loss is reasonable considering that the roundtrip includes 6 mirror-reflections, 8 slab-reflections, and 16 slab-air interfaces in addition to the end mirror losses. The material absorption and scattering loss was measured at AlliedSignal to be $0.15 \% / \mathrm{cm}$ and was therefore a minor component of the overall cavity loss.

The pumping efficiency can be obtained from the magnitudes of the thresholds measured here. The path length through the slab, for 4 bounces, is calculated to be $L=$ $1.53 \mathrm{~cm}$ which leads to a spatially-averaged, peak gain per pump Joule $\left[n^{*}\right] \sigma / P=0.0026 \mathrm{~cm}^{-1} \mathbf{J}^{-1}$. In this experiment, the electric field is polarized at $\theta_{i}=33^{\circ}$ to the crystalline $c$-axis and the effective emission cross section is given by $\sigma=\left(\sigma_{\pi}-\sigma_{\pi, \mathrm{ESA}}\right) \cos ^{2}\left(\theta_{i}\right)+\left(\sigma_{\sigma}-\sigma_{\sigma, \mathrm{ESA}}\right) \sin ^{2}\left(\theta_{i}\right)$. Excitedstate absorption reduces the $\pi$-polarized cross section at $\lambda=$ $850 \mathrm{~nm}$ from 4.8 to $3.0 \times 10^{-20} \mathrm{~cm}^{2}$ and the angle-dependent factors, including the negative contribution of $\sigma_{\sigma}-\sigma_{\sigma, \mathrm{ESA}}$, further reduces $\sigma$ to $2.0 \times 10^{-20} \mathrm{~cm}^{2}$ [9]. The gain calculated from these threshold measurements is, in fact, consistent with the single pass gain we measured directly in the earlier work [7]. There we obtained $g / P=0.0053 \mathrm{~cm}^{-1} \mathrm{~J}^{-1}$ for a similar $16 \%$ Cr-doped slab (1.77 mm thick) with $\mathrm{E} \| \mathrm{c}$. In the present work, the relevant cross section is reduced by $1 / 3$ and, in addition, the dielectric coating on the slab has a less than perfect transmission at the pump wavelengths. The average transmission of the coating was measured to be $\sim 80 \%$ in the red absorption band and $\sim 65 \%$ in the blue band. The stored energy density per pump Joule, given by $\left[n^{*}\right] h \nu_{L} / P$, is a measure of the energy storage efficiency and is calculated to be $0.03 \mathrm{~cm}^{-3}$ in this experiment compared to $0.04 \mathrm{~cm}^{-3}$ in the earlier work.

The slope efficiencies reported in Table I represent limiting values observed at the higher pump energies. The output was generally found to be linear in this region and no significant roll-off was observed. The slope efficiencies $\eta$ are considered to depend on the mirror transmission and cavity loss according to

$$
1 / \eta=1 / \eta_{o}[1+\ln (1-\alpha) / \ln (1-T)]
$$

from which we obtain $\alpha=0.048$ and an intrinsic slope efficiency $\eta_{o}=1.05 \mathrm{~mJ} / \mathrm{J}$. $\eta_{o}$ should be comparable to the energy storage efficiency, calculated from the threshold data above, times the effective extraction volume. An estimate for the largest possible extraction volume (limited by the width of the slab) would be $\sim 11.4 \times 1.6 \times 9=164 \mathrm{~mm}^{3}$ which gives a stored energy per pump Joule equal to $4.9 \mathrm{~mJ} / \mathrm{J}$ clearly greater than $\eta_{o}$. However, the strongest pumping was in the center of the slab, closest to the flash-lamp, and at these pump energies there was little extraction from the outside edges. At least two other phenomena have been identified which also contribute to the lower values of $\eta_{o}$ and $\alpha$ obtained here. As was mentioned earlier, upconversion loss from the $\mathrm{Cr}^{3+4} \mathrm{~T}_{2}$ state at the higher pump energies limit the slope efficiencies, especially for larger mirror output coupling, since the gain is then clamped at a higher value. Also, the flash-lamp pumping was found to be somewhat nonlinear with increasing energy. The waveform of the pump light transmitted through a Corning 4-64 green filter was measured for pump energies from 14 to $44 \mathrm{~J}$. This filter transmits from $480-560 \mathrm{~nm}$, midway between the two strong absorption features in $\mathrm{Cr}_{\mathrm{LiSrAlF}} 6$ which extend from $400-700 \mathrm{~nm}$. The pulse shape remained very nearly constant over this range. The peak intensity increased linearly with pump energy up to $\sim 30 \mathrm{~J}$, after which a slight roll-off was observed at higher energies, with the output at $44 \mathrm{~J}$ down $\sim 10 \%$ from the linear extrapolation. This is presumably due to saturation and/or the blue-shifting of the flash-lamp emission with increasing current density. Since the measured threshold energies were all well below $30 \mathrm{~J}$, they would not be affected by this.

The laser emission spectrum, recorded with a silicon detector array, is shown in Fig. 4. This data was measured with the $6.8 \%$ output mirror, pumping with $33 \mathrm{~J}$, and represents the average of several shots. There is a good deal of structure present due to interference effects in the laser cavity. The strong modulation at $1.3 \mathrm{~nm}$ is evidently due to the etalon effect between the bounce-mirror and the uncoated slab surface. This wavelength period is consistent with an incident angle of $50^{\circ}$ and a $0.44 \mathrm{~mm}$ spacing in air. A shorter wavelength period of $\sim 0.2 \mathrm{~nm}$, which is just barely resolved, is due to the interference effects between the top and bottom surfaces of the $1.6 \mathrm{~mm}$ slab. 


\section{THERMAL EFFECTS}

After several thousand flash-lamp pulses there was some noticeable deterioration of the cemented interface between the slab and the sapphire window. This was evident by slight interference bands over $\sim 20 \%$ of the total area. It was not clear if this was due to separation of the dielectric coating from the slab or of the slab from the sapphire. At this point, we thought it worthwhile to sacrifice the slab to a test of average power. The lamps were run at $38 \mathrm{~J}$ and the repetition rate was increased in small increments. Attempts were made to measure the laser power during this procedure, however the general heating of mirror mounts and air turbulence made this impractical. It should be emphasized here that the slab covered less than half of the lamp aperture and a large part of the flashlamp light was free to escape from the head. Reflective covering was purposely not used to block this output so that laser efficiency extrapolations could be made for the full aperture. The individual mirror mounts were however covered with aluminum foil which helped reduce heating effects.

One corner of the slab finally developed a crack at $40-45$ $\mathrm{Hz}$. The heat loading $Q(\mathrm{~J} / \mathrm{cc})$ in the slab can be estimated from the energy storage efficiency according to

$$
Q=h\left(\nu_{p}-\nu_{f}\right)\left[n^{*}\right] / \beta .
$$

Here,

$$
\begin{aligned}
\beta & =\max \left(\int_{o}^{t} p\left(t^{\prime}\right) \exp \left[-\left(t-t^{\prime}\right) / \tau\right] d t^{\prime}\right) / \int p(t) d t \\
& =0.56
\end{aligned}
$$

is obtained from the measured pump pulse wave form $p(t)$ and fluorescence lifetime $\tau$ and relates the peak inversion to the total pump energy. If we assume $\tau=60 \mu$ s for the $16 \%$ Cr-doped material [4] and that the effective pump and fluorescence wavelengths are 530 and $840 \mathrm{~nm}$, respectively, then we arrive at $Q d=5.1 \mathrm{~mJ} / \mathrm{cm}^{2}$ per Joule of pump. This results in a heat loading per unit area, at the extrapolated fracture conditions, of $\sim 8 \mathrm{~W} / \mathrm{cm}^{2}$. Since the nonradiative components of the fluorescence decay, such as thermal quenching and excited-state upconversion have been neglected, the actual heat loading at these conditions would be somewhat higher. The knowledge of the heat loading in the slab allows us to estimate the temperature rise in the slab under the variety of operating conditions in this experiment. The thermal conductivity in the direction perpendicular to the $c$-axis is required, however we must use the measured value [3] along the $c$-axis, $\kappa_{c}=0.031$ $\mathrm{W} / \mathrm{cm} /{ }^{\circ} \mathrm{C}$. Since the majority of heat is deposited close to the heat-sink, we can get a rough idea of the temperature rise $\Delta T$ across the slab, by considering the heat to flow across $\sim$ half the slab thickness. The maximum heat loading during the laser measurements $\left(4 \mathrm{~Hz}\right.$ and $44 \mathrm{~J}$ ) gives only $\Delta T=2.1^{\circ} \mathrm{C}$ which is not significant. However at the fracture conditions $\Delta T=21^{\circ} \mathrm{C}$, and with the additional temperature drop across the window and cement bond, the outside of the slab would exhibit a significant reduction in fluorescence lifetime [10].

\section{SUMMARY}

In summary, the performance of a conductively-cooled, multi-bounce Cr:LiSAF slab-laser has been characterized. A thin, highly doped slab of Cr:LiSAF was cemented onto a sapphire window which served both as a heat-sink and a transparent window for the flash-lamp pump light. The active portion of the slab overlapped $\sim 15 \%$ of the length of the lamp along one side and a maximum laser energy of $9 \mathrm{~mJ}$ was obtained with a slope efficiency of $0.609 \mathrm{~mJ} / \mathrm{J}$. The round-trip cavity loss was determined to be $8 \%$ due to the multiple reflections and interfaces in the cavity. If this loss could be reduced by improving the transmission and reflection characteristics of the dielectric coating, a factor-oftwo improvement in the efficiency could probably be realized. Also, the output pulse energy could potentially be scaled by $\sim 10 \times$ if the full pump length of both sides of the lamp was used. In order to obtain higher average power by running the laser closer to the observed fracture limit, more careful attention should be paid to the choice of adhesive used to bond the slab to the sapphire window. The sapphire does provide a rigid and transparent support for the slab and should help to mitigate the problem of thermally induced bowing that might occur with an unsupported water cooled slab.

\section{ACKNOWLEDGMENT}

The author would like to thank R. Morris and co-workers at Allied-Signal Inc. for their efforts in growing and supplying the high-quality Cr:LiSAF material, and H. Rieger of W. J. Schafer Associates, Inc., for several helpful discussions.

\section{REFERENCES}

[1] M. Stadler, B. H. T. Chai, and M. Bass, "Flashlamp pumped Cr:LiSrAlF 6 laser," Appl. Phys. Lett., vol. 58, pp. 216-218, 1991.

[2] T. Ditmire and M. D. Perry, "Terawatt Cr:LiSrAlF 6 laser system," Opt. Lett., vol. 18 , pp. 426-428, 1993.

[3] S. A. Payne, L. K. Smith, R. J. Beach, B. H. T. Chai, J. B. Tassano, L D. DeLoach, W. L. Kway, R. W. Solarz, and W. F. Krupke, "Properties of Cr:LiSrAlF 6 crystals for laser operation," submitted to Appl. Opt.

[4] S. A. Payne, W. F. Krupke, L. K. Smith, W. L. Kway, L. D. DeLoach, and J. B. Tassano, "752 nm wing-pumped Cr:LiSAF laser," IEEE $J$ Quantum Electron., vol. 28, pp. 1188-1196, 1992.

[5] L. K. Smith, S. A. Payne, W. F. Krupke, L. D. DeLoach, R. Morris, E W. O'Dell, and D. J. Nelson, "Laser emission from the transition-metal compount $\mathrm{LiSrCrF}_{6}, "$ Opt. Lett., vol. 18, pp. 200-202, 1993.

[6] J. L. Emmett, W. F. Krupke, and W. R. Sooy, "The potential of high-average power solid state lasers," LLNL Rep. UCRL-53571, 1984

[7] F. Hanson, C. Bendall, and P. Poirier, "Gain measurements and average power capabilities of $\mathrm{Cr}^{3+}: \mathrm{LiSrAlF}_{6}, "$ Opt. Lett., vol. 18, pp. $1423-1425,1993$.

[8] M. A. Nogonov, H. P. Jenssen, and A. Cassanho, Advanced Solid-State Lasers and Compact Blue-Green Lasers Technical Digest, Washington, DC: Optical Society of America, 1993, paper ATuF4.

[9] W. R. Rappaport, "Higher average power operation of Cr:LiSAF and Cr:LiSGAF pumped by alexandrite," Proc. OSA, Advanced Solid-State Lasers and Compact Blue-Green Lasers, Lloyd Chase and Albert Pinto, Eds. Washington, DC: Optical Society of America, 1993, vol. 2.

[10] M. Stalder, M. Bass, and B. H. T. Chai, "Thermal quenching of fluorescence in chromium-doped fluoride laser crystals," J. Opt. Soc. of Am. B, vol. 9, pp. 2271-2273, 1992.

F. Hanson, photograph and biography not available at the time of publication. 